\title{
On Variations of the Brightness of Type la Supernovae With the Age of the Host Stellar Population
}

Brendan K. Krueger*

Stony Brook University

E-mail: brendan.krueger@stonybrook . edu

Aaron P. Jackson

Stony Brook University

Alan C. Calder

Stony Brook University

Dean M. Townsley

University of Alabama

Edward F. Brown

Michigan State University

\section{F. X. Timmes}

\section{Arizona State University}

Recent observational studies of type Ia supernovae (SNeIa) suggest correlations between the brightness of an event and properties of the host galaxy that appear to involve the age of the progenitor population. One way to influence the explosion systematically is through the central density at ignition, which is determined by the mass of the white dwarf before the onset of accretion, the white dwarf cooling time (prior to the onset of accretion), the subsequent accretion history, and neutrino losses. The dependence of the central density on cooling time connects the central density to the age of the progenitor and therefore the average stellar age of the host galaxy. We find that with increased progenitor central density, production of Fe-group material does not change but production of ${ }^{56} \mathrm{Ni}$ decreases, which we attribute to a higher rate of neutronization occurring at higher density. These results offer an explanation for the observation of dimmer SNeIa in galaxies with an older stellar population. We also demonstrate a strong dependence of the ${ }^{56} \mathrm{Ni}$ yield in our results on the morphological structure of the burning front during the early deflagration, suggesting that a statistical ensemble of simulations is necessary when studying the systematics of SNeIa.

11th Symposium on Nuclei in the Cosmos, NIC XI

July 19-23, 2010

Heidelberg, Germany

${ }^{*}$ Speaker. 


\section{Introduction}

The most widely accepted setting for a type Ia supernova explosion is a thermonuclear runaway occurring in a $\mathrm{C} / \mathrm{O}$ white dwarf (WD) that has gained mass from a stellar companion [1]. In this "single degenerate" scenario, the WD gains mass, compressing and heating the core until a flame is born that rapidly consumes the white dwarf. We explore the systematics of these events with models that assume this paradigm. Specifically, our models assume that the explosion begins with a subsonic deflagration born in the interior of the WD that transitions to a supersonic detonation wave that rapidly incinerates the star, the deflagration-to-detonation transition (DDT) paradigm [2]. We utilize a theoretical framework for statistical studies [3] using two-dimensional simulations with a customized version of the Flash code developed at the University of Chicago [4, 5, 6, 7].

\section{The Role of Central Density}

After forming, the progenitor WD is isolated from any significant source of heat input and cools. Once accretion begins, the WD core temperature increases. An initially cooler WD requires a higher central density for accretion to heat the WD core to the temperature at which carbon burning ignites [8] (See also [9]). SNeIa are observed in a surrounding stellar population, the age of which is defined as the time since the last star-forming event. A SNIa from an older stellar population may have undergone a longer period of isolation, leading to a higher central density. Thus we study the effect of central density on ${ }^{56} \mathrm{Ni}$ yield as a proxy for a relation between age of the stellar population and mean brightness of SNeIa.

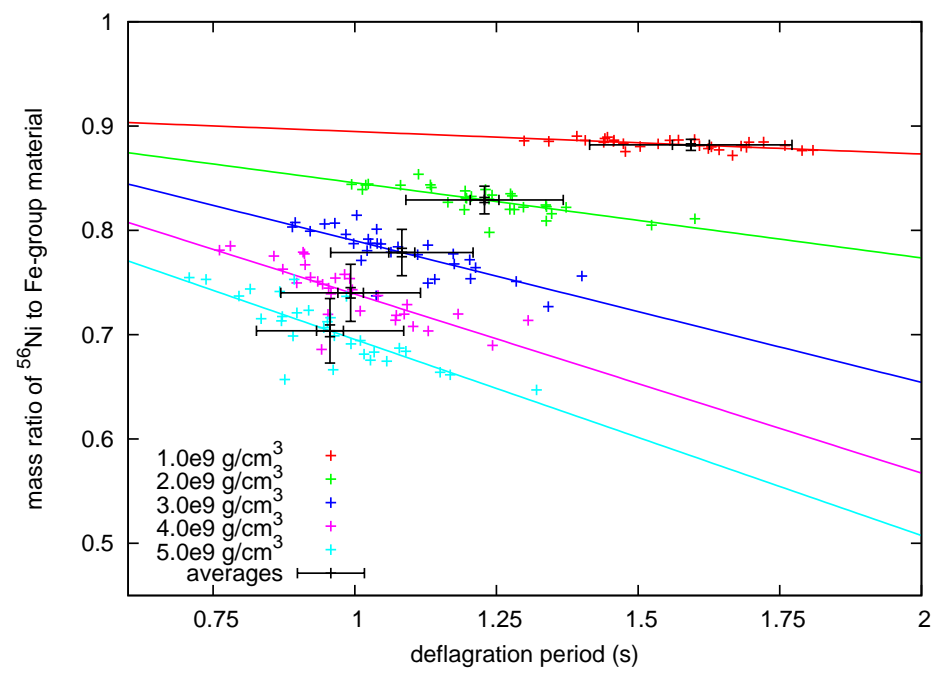

Figure 1: Plot of the ${ }^{56} \mathrm{Ni}$-to-Fe-group mass ratio vs. duration of the deflagration (subsonic burning) phase, with realizations grouped by central density of the progenitor. For a given progenitor, more time spent in subsonic burning allows a greater degree of neutronization, thus lowering the yield of ${ }^{56} \mathrm{Ni}$. Additionally, despite occurring faster, SNeIa from progenitors with a higher central density undergo a higher degree of neutronization so that the yield of ${ }^{56} \mathrm{Ni}$ decreases despite nearly constant production of Fe-group material.

Our progenitors are WD stars with convective, carbon-depleted cores and isothermal envelopes. We constructed five progenitors to study the effect of progenitor central density on ${ }^{56} \mathrm{Ni}$ 
yield, and therefore the brightness of the SNIa. We initiate burning with a "match head" of burned material at the center of the star perturbed by spherical harmonics. Each perturbation spectrum ("realization") is generated by a seed number; we simulate 30 independent realizations for each progenitor to generate a population of SNeIa at each density, giving an idea of the intrinsic statistical spread of SNeIa in addition to the variation due to progenitor central density.

\section{Results}

We find that as the central density of the progenitor increases the amount of radioactive ${ }^{56} \mathrm{Ni}$ produced decreases; quantitatively, we find that an increase of $10^{9} \mathrm{~g} \mathrm{~cm}^{-3}$ in central density lowers the ${ }^{56} \mathrm{Ni}$ yield by $0.047 \pm 0.003 M_{\odot}$. This is the result of a higher rate of neutronization at higher densities, which pushes the nucleosynthetic yields towards more neutron-rich, stable isotopes such as ${ }^{58} \mathrm{Ni}$. The radioactive ${ }^{56} \mathrm{Ni}$ powers the light curve of the SNIa, so a lower ${ }^{56} \mathrm{Ni}$ yield directly implies a dimmer SNIa. Recalling our tentative identification of central density as a proxy for the age of the stellar population, these two correlations show that SNeIa from older stellar populations are predicted to appear dimmer. This is in agreement with recent observations $[10,11,12,13]$.

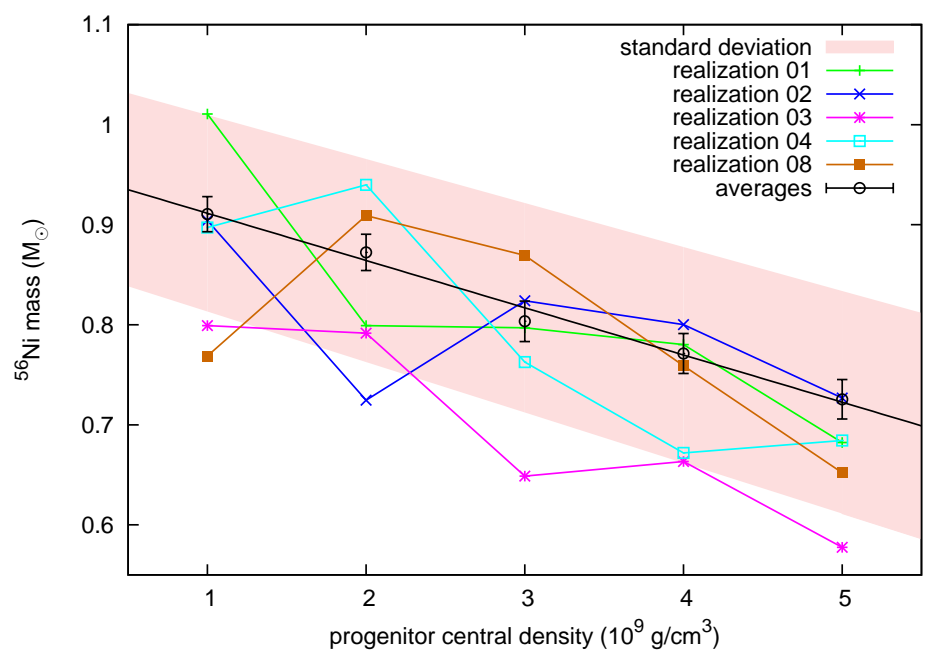

Figure 2: Plot of ${ }^{56} \mathrm{Ni}$ vs. progenitor central density for five realizations, illustrating the variety of trends that can be seen within a realization. These include non-monotonic trends suggesting an increase of ${ }^{56} \mathrm{Ni}$ with central density instead of the decrease seen in a statistical ensemble.

As we vary the central density of the progenitor within a realization, we find a considerable scatter with most cases showing non-monotonic ${ }^{56} \mathrm{Ni}$ yield. Any observed trend in a single realization could thus be deceiving. We believe the scatter to follow from a high morphological dependence, which varies the time spent in the deflagration stage and strongly impacts the trend in ${ }^{56} \mathrm{Ni}$. Because of this effect, any study of trends in SNeIa should consider the statistics of a suite of simulations in order to capture the actual behavior of SNeIa.

Höflich et al. (2010) [14] argue that ${ }^{56} \mathrm{Ni}$ in the central regions of the exploding WD does not contribute to the light curve at maximum, and therefore they do not see a significant trend with central density at maximum brightness, but in late-time brightness. The pure-deflagration models of 
Röpke et al. (2006) [15] exhibit a shallow increase of produced ${ }^{56} \mathrm{Ni}$ as central density increases, in contradiction of our findings. Iwamoto et al. (1999) [16] find that the trend with central density depends on the DDT transition density; extrapolating from their results, our value of transition density should yield an increasing ${ }^{56} \mathrm{Ni}$ yield as central density increases. Bravo et al. $(1993,1996)[17,18]$ find a similar effect of lowering ${ }^{56} \mathrm{Ni}$ fraction due to increasing electron capture. But since more mass burns, they find that the peak brightness is not strongly dependent upon the central density, in contrast to our results. Complete details of our study may be found in Krueger et al. (2010) [19].

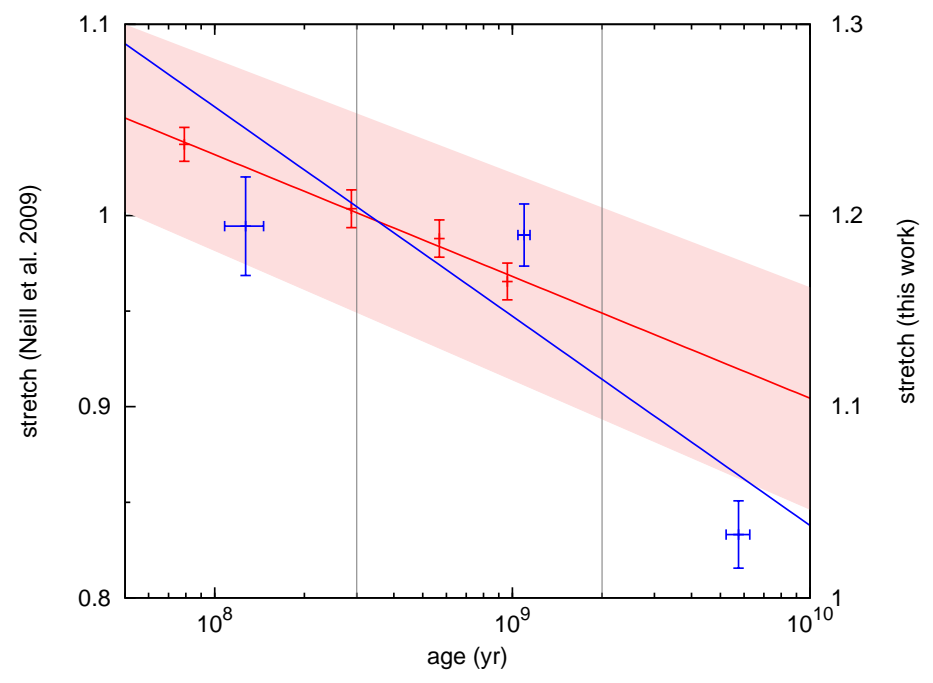

Figure 3: Plot of stretch vs. age. In blue are the results from Neill et al. (2009) [12]; the vertical grey lines show where they binned and averaged their data. In red are the results from this work converted to age [8] and stretch [20]; error bars for our results show the standard error of the mean and the shaded region shows the standard deviation. Our models systematically overproduce ${ }^{56} \mathrm{Ni}$ and the central density-age conversion has large uncertainties; to account for this, our data is plotted on a shifted scale (shown on the right axis) in order to compare the trends. Our results show a declining stretch with increasing age, as seen in observations, but with a shallower slope, suggesting the central density effect contributes to this observational finding.

\section{Acknowledgements}

This work was supported by the Department of Energy through grants DE-FG02-07ER41516, DE-FG02-08ER41570, and DE-FG02-08ER41565, and by NASA through grant NNX09AD19G. A.C.C. acknowledges support from the Department of Energy under grant DE-FG02-87ER40317. D.M.T. received support from the Bart J. Bok fellowship at the University of Arizona for part of this work. The authors gratefully acknowledge the generous assistance of Pierre Lesaffre, as well as fruitful discussions with Mike Zingale, and the use of NSE and weak reaction tables developed by Ivo Seitenzahl. The authors also acknowledge the hospitality of the KITP, which is supported by NSF grant PHY05-51164, during the programs "Accretion and Explosion: the Astrophysics of Degenerate Stars" and "Stellar Death and Supernovae." The software used in this work was in part developed by the DOE-supported ASC/Alliances Center for Astrophysical Thermonuclear Flashes at the University of Chicago. This research utilized resources at the New York Center for Computational Sciences at Stony Brook University/Brookhaven National Laboratory which is 
supported by the U.S. Department of Energy under contract no. DE-AC02-98CH10886 and by the State of New York.

\section{References}

[1] W. Hillebrandt and J. C. Niemeyer. Type Ia supernova explosion models. Annu. Rev. Astron. Astrophys, 38:191, 2000.

[2] A. M. Khokhlov. Delayed detonation model for type Ia supernovae. Astronomy and Astrophysics, 245:114-128, 1991

[3] D. M. Townsley et al. Evaluating Systematic Dependencies of Type Ia Supernovae: The Influence of Progenitor ${ }^{22} \mathrm{Ne}$ Content on Dynamics. Astrophysical Journal, 701:1582-1604, August 2009.

[4] B. Fryxell et al. FLASH: An adaptive mesh hydrodynamics code for modeling astrophysical thermonuclear flashes. Astrophysical Journal Supplement, 131:273-334, 2000.

[5] A. C. Calder et al. On validating an astrophysical simulation code. Astrophysical Journal Supplement, 143:201-229, 2002.

[6] A. C. Calder et al. Capturing the Fire: Flame Energetics and Neutronization for Type Ia Supernova Simulations. Astrophysical Journal, 656:313-332, February 2007.

[7] D. M. Townsley et al. Flame Evolution During Type Ia Supernovae and the Deflagration Phase in the Gravitationally Confined Detonation Scenario. Astrophysical Journal, 668:1118-1131, October 2007.

[8] P. Lesaffre et al. The C flash and the ignition conditions of Type Ia supernovae. Monthly Notices of the Royal Astronomical Society, 368:187-195, May 2006.

[9] E. García-Berro et al. A white dwarf cooling age of 8Gyr for NGC 6791 from physical separation processes. Nature, 465:194-196, May 2010.

[10] F. Mannucci, M. Della Valle, and N. Panagia. Two populations of progenitors for Type Ia supernovae? Monthly Notices of the Royal Astronomical Society, 370:773-783, August 2006.

[11] J. S. Gallagher et al. Supernovae in Early-Type Galaxies: Directly Connecting Age and Metallicity with Type Ia Luminosity. Astrophysical Journal, 685:752-766, October 2008.

[12] J. D. Neill et al. The Local Hosts of Type Ia Supernovae. Astrophysical Journal, 707:1449-1465, December 2009.

[13] M. L. Graham et al. The Type Ia Supernova Rate in Radio and Infrared Galaxies from the Canada-France-Hawaii Telescope Supernova Legacy Survey. Astronomical Journal, 139:594-605, February 2010.

[14] P. Höflich et al. Secondary Parameters of Type Ia Supernova Light Curves. Astrophysical Journal, 710:444-455, February 2010.

[15] F. K. Röpke. Multi-dimensional numerical simulations of type Ia supernova explosions (With 7 Figures). In S. Roeser, editor, Reviews in Modern Astronomy, volume 19 of Reviews in Modern Astronomy, pages 127-+, January 2006.

[16] K. Iwamoto et al. Nucleosynthesis in Chandrasekhar mass models for type Ia supernovae and constraints on progenitor systems and burning-front propagation. Astrophysical Journal Supplement, 125:439-462, 1999 
[17] E. Bravo et al. On the photometric homogeneity of Type IA supernovae. Astronomy and Astrophysics, 269:187-194, 1993.

[18] E. Bravo et al. Clues to Type IA SN progenitors from degenerate carbon ignition models. Astronomy and Astrophysics, 306:811-+, 1996.

[19] B. K. Krueger et al. On Variations of the Brightness of Type Ia Supernovae with the Age of the Host Stellar Population. Astrophysical Journal Letters, 719:L5-L9, August 2010.

[20] D. A. Howell et al. The Effect of Progenitor Age and Metallicity on Luminosity and ${ }^{56} \mathrm{Ni}$ Yield in Type Ia Supernovae. Astrophysical Journal, 691:661-671, January 2009. 